\title{
RIP-ed and ready to dance: new mechanisms for polycystin-1 signaling
}

\author{
Lisa M. Guay-Woodford
}

Departments of Medicine, Genetics, and Pediatrics, University of Alabama at Birmingham, Birmingham, Alabama, USA.

\begin{abstract}
Polycystin-1, the protein encoded by the principal gene involved in autosomal dominant polycystic kidney disease, has been implicated in extracellular sensing as well as in cell-cell and cell-matrix interactions. However, the precise mechanisms involved in polycystin-1 signaling are not well defined. A report in this issue of the JCI demonstrates that the C-terminal tail of polycystin- 1 is cleaved from the membrane through regulated intramembrane proteolysis (RIP) and that this domain translocates to the nucleus, where it activates the AP-1 transcription pathway (see the related article beginning on page 1433). This translocation appears to be modulated by polycystin-2, with which polycystin-1 is thought to interact. These findings provide what we believe to be the first evidence that polycystin-1 can signal directly to the nucleus and that polycystin-1-polycystin- 2 interactions do not require colocalization of these proteins in the same membrane compartment.
\end{abstract}

Autosomal dominant polycystic kidney disease (ADPKD) occurs in 1 in 500-1,000 individuals. This slowly progressive disorder results from mutations in 1 of 2 genes, $P K D 1$ or $P K D 2$, which encode the proteins polycystin-1 and polycystin-2, respectively (1). Polycystin- 1 is a $462-\mathrm{kDa}$ cell membrane-associated glycoprotein with a number of putative adhesion domains and a $G$ protein-coupled receptor proteolytic site (GPS) within its amino-terminus; 7-11 transmembrane (TM) spanning regions; and a 200-AA intracellular C-terminus that includes a coiled-coil domain that mediates binding to polycystin-2 (reviewed in ref. 2). Polycystin-2 is also predicted to be an integral membrane protein of $110 \mathrm{kDa}$ with $6 \mathrm{TM}$ domains and intracellular $\mathrm{N}$ - and C-termini. The C-terminus contains an incomplete coiled-coil domain that is involved in binding to polycystin-1 and an EF-hand domain that is predicted to have calcium-binding and regulatory functions (3).

Nonstandard abbreviations used: ADPKD, autosomal dominant polycystic kidney disease; CTT, C-terminal tail; GPS, G protein-coupled receptor proteolytic site; RIP, regulated intramembrane proteolysis; TM, transmembrane; TRP, transient receptor potential; UUO, unilateral ureteral obstruction.

Conflict of interest: The author has declared that no conflict of interest exists.

Citation for this article: J. Clin. Invest. 114:1404-1406 (2004). doi:10.1172/JCI200423544.

\section{Polycystin-1-polycystin-2 interactions and intracellular signaling pathways}

Several lines of evidence indicate that polycystin- 1 and polycystin- 2 physically interact and the polycystin-1-polycystin-2 complex is involved in tubular patterning signaling pathways (4), with polycystin-1 acting as a sensor of extracellular signals and polycystin-2 assembling as either a homotetramer or heterotetramer with transient receptor potential (TRP) channels to function as a regulated ion channel (5). For example, recent studies from the Zhou laboratory and others have shown that the polycystins are expressed in the primary apical cilia, where the polycystin-1-polycystin-2 protein complex appears to function as a mechanosensor, detecting cilial movement and transducing the signal through intracellular calcium transients (6-8). The Walz group has demonstrated that overexpression of the polycystin-1 C-terminus in cultured renal epithelia induces branching morphogenesis through a PKC-dependent effector pathway that appears to require polycystin-2 interaction with the polycystin-1 coiled-coil domain (9). In other studies, this group has demonstrated that polycystin-2 and the polycystin-1 C-terminus act synergistically through heterotrimeric $\mathrm{G}$ proteins to mediate PKC- $\alpha-$ dependent and JNK-dependent activation of the transcription factor AP-1 $(10,11)$. In addition, the polycystin-1 C-terminal domain appears to modulate the Wnt signal- ing pathway by stabilizing levels of cytosolic $\beta$-catenin (12). Moreover, the Germino laboratory has determined that polycystin-1 can physically bind and activate JAK2 kinase in a polycystin-2-dependent manner, leading to the activation of STAT1. Activated STAT1 homodimerizes, translocates to the nucleus, and transcriptionally upregulates the cyclindependent kinase inhibitor p21(cip1/waf1) (13), which in turn causes cells to exit the cell cycle and become terminally differentiated.

By and large, these findings presuppose that polycystin-1 and polycystin-2 physically interact to initiate kinase/ phosphatase-mediated signaling cascades. However, recent reports indicate that the subcellular distribution of these proteins is not entirely coincident. While both polycystin-1 and polycystin-2 have been localized to the primary apical cilium, polycystin-1 has also been localized to numerous other membrane domains, including the apical plasma membrane, tight junctions, adherens junctions, desmosomes, and focal adhesion plaques. In contrast, polycystin-2 is thought to be either targeted to the endoplasmic reticulum or distributed to the plasma membrane as well as the endoplasmic reticulum (reviewed in ref. 1). This complex pattern of subcellular localizations begs the question; how can polycystin-1 and polycystin-2 interact to effect intracellular signaling when the proteins are often not expressed in the same subcellular compartment? The report by Chauvet et al. (14) in this issue of the JCI provides the first evidence that the C-terminal tail of polycystin-1 can be proteolytically released from the membrane and translocated to the nucleus in a process that is modulated by polycystin-1-polycystin-2 interaction.

\section{Regulated intramembrane proteolysis modulates the release of membrane-bound transcription factors}

Chauvet et al. (14) demonstrate that in renal epithelial cells overexpressing fulllength polycystin-1, the C-terminal tail 


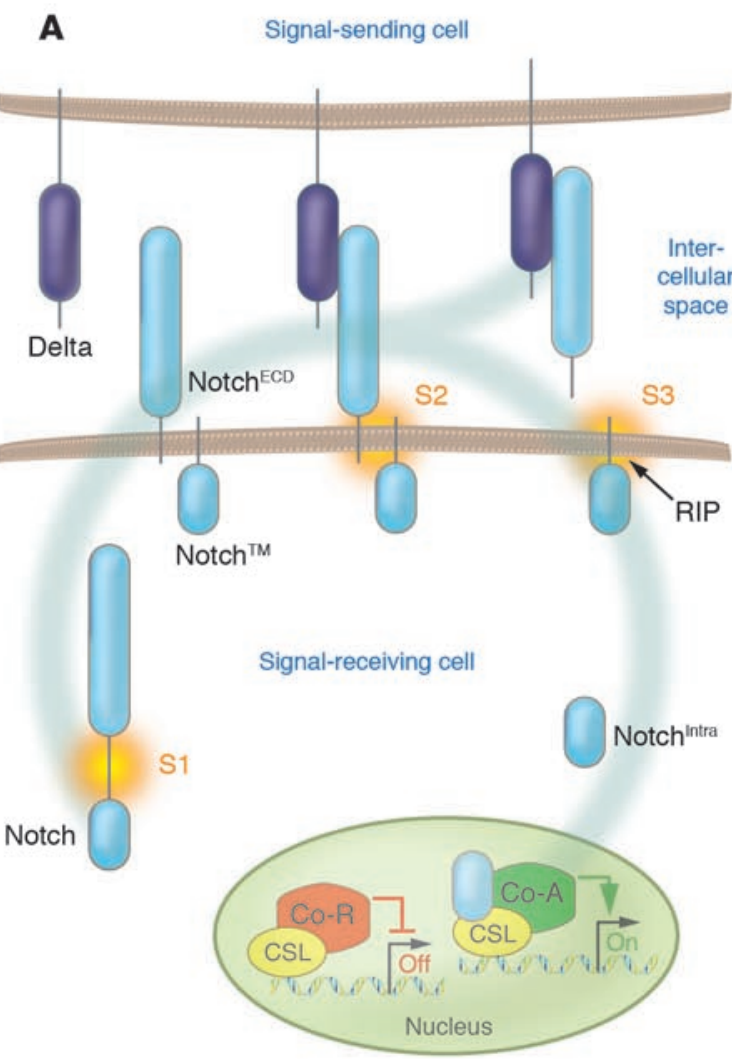

B

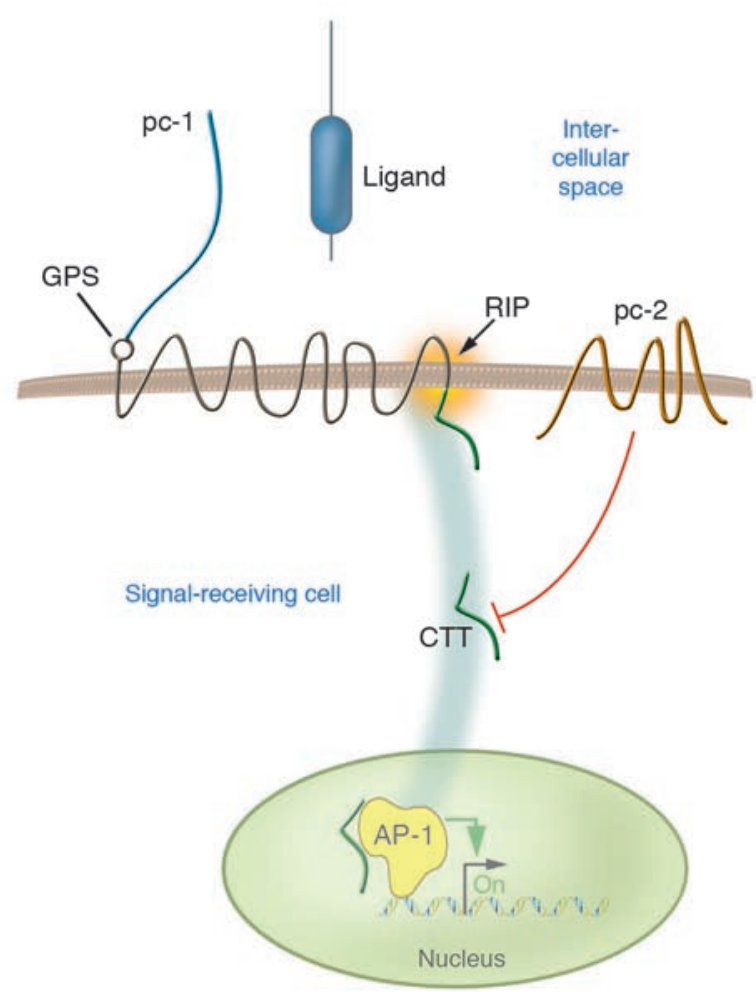

\section{Figure 1}

Models of Notch and polycystin-1 signaling via RIP. (A) Newly synthesized Notch is constitutively cleaved in the trans-Golgi network by furin-like proteases. Following this site 1 (S1) cleavage event, the bipartite Notch receptor consisting of a noncovalent interaction between the ectodomain (Notch ${ }^{C D}$ ) and a membrane-tethered intracellular domain (Notch ${ }^{\mathrm{TM}}$ ) is inserted into the plasma membrane. Activation of Notch by its ligand, e.g., Delta, triggers 2 additional proteolytic events. S2 cleavage by extracellular proteases of the ADAM/TACE (a disintegrin and metalloproteinase/ TNF- $\alpha$-converting enzyme) family releases Notch $\mathrm{ECD}$ and generates an activated, membrane-bound form of Notch ${ }^{\mathrm{TM}}$ that is further processed via RIP-mediated S3 cleavage. These events lead to release of the Notch intracellular domain (Notchintra), which translocates into the nucleus and displaces the corepressor (Co-R) complex from the CBF-1, Su(H), Lag-1-type (CSL-type) transcription factors. The coactivating (Co-A) complex containing Notch Intra and CSL stimulates expression of CSL/Notch target genes. Figure modified from Development (17) with permission from the Company of Biologists Ltd.; and from Current Biology (18) with permission from Elsevier. (B) Polycystin-1 (pc-1) is a cell-surface receptor that undergoes regulated extracellular proteolytic processing at its GPS, which results in the release of its N-terminal fragment. In this issue of the $\mathrm{JCl}$, Chauvet et al. (14) show that the CTT of polycystin-1 can also be cleaved from its transmembrane anchor, presumably through RIP-related mechanisms. Once released, this domain translocates to the nucleus, where it activates the AP-1 transcription pathway. This translocation event appears to be regulated by polycystin-2.

(CTT) is cleaved from the membrane and targeted to the nucleus. Although the precise mechanism remains to be defined, the authors proposed that C-terminal cleavage occurs through a 2 -step mechanism called regulated intramembrane proteolysis (RIP), a conserved cellular process by which cells can transmit signals from one compartment to another through the liberation of membranebound transcription factors $(15,16)$. A number of cell surface growth factor receptors are subject to RIP after ligand binding and/or ectodomain cleavage.

A particularly illustrative paradigm involves Notch-mediated cell-cell signaling, a critical pathway involved in meta- zoan cell fate determination and pattern formation $(17,18)$. As diagrammed in Figure 1A, the Notch receptor is processed in the trans-Golgi network to a heterodimer before being expressed on the plasma membrane. Ligand-induced activation sensitizes the membrane-tethered intracellular domain to cleavage by extracellular proteases, thereby releasing the Notch ectodomain and generating an activated, membrane-bound truncation product that undergoes 2 RIP-mediated proteolytic events. This processing releases the Notch intracellular domain, which then translocates from the cytosol to the nucleus and activates target gene transcription. Thus, the Notch receptor is a membrane-bound transcription factor whose release to the nucleus is tightly regulated by RIP.

To a certain extent, polycystin-1 processing parallels that of Notch (Figure 1B). As noted, proteolytic cleavage at the GPS removes the N-terminal ectodomain of polycystin-1, and RIP-mediated mechanisms are proposed to release the CTT. At this point, it is not clear whether ligand binding and/or N-terminal cleavage precedes and is a prerequisite for the C-terminal cleavage, as is the case for Notch and many other RIP-processed receptors. Studies with Notch indicate that exceedingly small, histochemically undetectable amounts of intracellular 
Notch are sufficient for target gene activation (reviewed in ref. 17), and this may also apply in the case of the polycystin-1 CTT, as Chauvet et al. could not detect CTT-specific immunoreactivity in nuclear fractions prepared from wild-type mouse kidneys (14).

Chauvet et al. (14) demonstrate that in cell culture systems, a soluble construct containing the last 200 AAs of polycystin-1 (p200) includes a discrete localization signal that targets it to the nucleus, where it activates AP-1 reporter gene expression. This translocation event appears to be buffered by polycystin-2, as coexpression of polycystin-2 prevents nuclear localization of p200 and leads to a 2- to 3-fold reduction in AP-1 activation. The effect is abrogated in cell lines cotransfected with truncated polycystin-2 constructs that lack the polycystin-1-interacting domain. Thus, these data define a mechanism through which polycystin-2 can modulate polycystin-1 signaling independently of whether these proteins are targeted to the same membrane compartment.

\section{Do mechanical stimuli induce CTT cleavage and nuclear translocation?}

Polycystin-1 and polycystin-2 localize to the primary apical cilium and appear to function in a mechanosensory transduction pathway that responds to tubular flow. Therefore, the authors examined CTT nuclear localization in 2 in vivo mouse models in which flow-mediated mechanical stimulation of tubular epithelia was disrupted - unilateral ureteral obstruction (UUO) and kidney-specific inactivation of the cilial molecular motor protein kinesin 3a. Nuclear CTT was detected in both models but not in control animals, which prompted the authors to suggest that alterations in tubular flow or in the capacity of tubular epithelia to sense mechanical stimuli triggered an increase in polycystin-1 cleavage events (14). While the data are permissive for this interpretation, it remains highly speculative to suggest that the nuclear translocation of the polycystin-1 CTT represents the cellular response to the mechanical alterations caused by UUO. Ureteral obstruction may prompt many cellular and tubular events, in addition to reduction of tubular flow (reviewed in ref. 19). These include alterations in apoptosis, injury-induced pathways, and even modifications in epithelial differentiation that may be associated with changes in the primary cilium. Therefore, more directed experiments, such as those performed by Praetorius and Spring (20) are required to determine whether RIPmediated cleavage of polycystin-1 defines an alternative cilial transduction pathway that is initiated in response to mechanical stimuli.

\section{RIP and signaling crosstalk}

Taken together, the findings presented by Chauvet et al. (14) make a compelling case that RIP and CTT nuclear translocation are critical events in polycystin-1-mediated intracellular signaling. Furthermore, the interactions between polycystin-1 and several other signaling pathways, including Wnt, JNK/AP-1, and JAK/STAT, are effected primarily through the polycystin-1 carboxy terminal tail. These pathways play critical roles in cell differentiation, tubulogenesis, and apoptosis - processes that are disrupted in ADPKD. Therefore, it is tempting to speculate that once released from the membrane, the polycystin-1 CTT not only translocates to the nucleus to regulate target gene expression, but also functions in cytosolic compartments to integrate crosstalk among several pathways that are essential in tubular patterning. Since these actions are buffered by polycystin-2, the polycystins appear to act together in highly choreographed integration of signaling pathways that defines and maintains normal renal tubular architecture. Chauvet et al. provide what we believe to be the first evidence that this choreography is not restricted to the plasma membrane stage.

\section{Acknowledgments}

The author thanks Bradley Yoder for helpful comments on this article.

Address correspondence to: Lisa M. GuayWoodford, Division of Genetic and Translational Medicine, University of Alabama at Birmingham, 740 Kaul Human Genetics Building, 720 20th Street South, Birmingham, Alabama 35294-0005, USA. Phone:
(205) 934-7308; Fax: (205) 975-5689; E-mail: lgw@uab.edu.

1. Wilson, P. 2004. Polycystic kidney disease. N. Engl. J. Med. 350:151-164.

2. Igarashi, P., and Somlo, S. 2002. Genetics and pathogenesis of polycystic kidney disease. J. Am. Soc. Nephrol. 13:2384-2398.

3. Mochizuki, T., et al. 1996. PKD2, a gene for polycystic kidney disease that encodes an integral membrane protein. Science. 272:1339-1342.

4. Boletta, A., and Germino, G. 2003. Role of polycystins in renal tubulogenesis. Trends Cell Biol. 13:484-492.

5. Somlo, S., and Ehrlich, B. 2001. Human disease: calcium signaling in polycystic kidney disease. Curr. Biol. 11:356-360.

6. Yoder, B.K., Hou, X., and Guay-Woodford, L.M. 2002. The polycystic kidney disease proteins, polycystin-1, polycystin-2, polaris, and cystin, are co-localized in renal cilia. J. Am. Soc. Nephrol. 13:2508-2516.

7. Nauli, S., et al. 2003. Polycystins 1 and 2 mediate mechanosensation in the primary cilium of kidney cells. Nat. Genet. 33:129-137.

8. Pazour, G., and Witman, G. 2003. The vertebrate primary cilium is a sensory organelle. Curr. Opin. Cell Biol. 15:105-110.

9. Nickel, C., et al. 2002. The polycystin-1 C-terminal fragment triggers branching morphogenesis and migration of tubular kidney epithelial cells. J. Clin. Invest. 109:481-489. doi:10.1172/ JCI200212867.

10. Arnould, T., et al. 1998. The polycystic kidney disease 1 gene product mediates protein kinase $\mathrm{C}$ alpha-dependent and c-Jun N-terminal kinasedependent activation of the transcription factor AP-1. J. Biol. Chem. 13:6013-6018.

11. Arnould, T., et al. 1999. Cellular activation triggered by the autosomal dominant polycystic kidney disease gene product PKD2. Mol. Cell. Biol. 19:3423-3434.

12. Kim, E., et al. 1999. The polycystic kidney disease 1 gene product modulated Wnt signaling. J. Biol. Chem. 274:4947-4953.

13. Bhunia, A., et al. 2002. PKD1 induces p21(waf1) and regulation of the cell cycle via direct activation of the JAK-STAT signaling pathway in a process requiring PKD2. Cell. 109:157-168.

14. Chauvet, V., et al. 2004. Mechanical stimuli induce cleavage and nuclear translocation of the polycystin-1 C-terminus. J. Clin. Invest. 114:1433-1443. doi:10.1172/JCI200421753.

15. Urban, S., and Freeman, M. 2002. Intramembrane proteolysis controls diverse signalling pathways throughout evolution. Curr. Opin. Genet. Devel. 12:512-518.

16. Landman, N., and Kim, T. 2004. Got RIP? Presenilin-dependent intramembrane proteolysis in growth factor receptor signaling. Cytokine Growth Factor Rev. 15:337-351.

17. Lai, E. 2004. Notch signaling: control of cell communication and cell fate. Development. 131:965-973.

18. Schweisguth, F. 2004. Notch signaling activity. Curr. Biol. 14:R129-R138.

19. Chevalier, R. 1999. Molecular and cellular pathophysiology of obstructive nephropathy. Pediatr. Nephrol. 13:612-619.

20. Praetorius, H., and Spring, K. 2001. Bending the MDCK cell primary cilium increases intracellular calcium. J. Membr. Biol. 184:71-79. 